\title{
Effect of atorvastatin in patients with chronic heart failure - insights from randomized clinical trials
}

Minwen Xu, Gaohui Yuan, Fanping Wei

Department of Cardiology, Huzhou Central Hospital, Huzhou, Zhejiang, China

Submitted: 24 July 2010

Accepted: 21 August 2010

Arch Med Sci 2010; 6, 6: 866-873

DOI: 10.5114/aoms.2010.19293

Copyright (c) 2010 Termedia \& Banach

\section{Abstract}

Introduction: Recent large clinical trials have yielded disappointing results of rosuvastatin in the chronic heart failure (CHF) population. The question that remains is whether these results of rosuvastatin studies could be extended to other statins. Therefore, we performed a meta-analysis based on all currently available randomized controlled trials (RCTs) to evaluate the clinical efficacy of atorvastatin in CHF patients.

Material and methods: The published literature was scanned by formal searches of electronic databases up to January 2010. RCTs were eligible for inclusion if they compared atorvastatin versus placebo treatment in patients with $\mathrm{CHF}$ and reported the clinical outcomes.

Results: Pre-specified criteria were met by 7 trials involving 540 patients. The primary endpoint, all-cause mortality, was significantly reduced with atorvastatin therapy compared with placebo in CHF patients (odds ratio [OR] 0.39, $P=0.002$ ), with similar results in cardiovascular mortality (OR $0.28, P=0.002)$ and sudden cardiac death (OR 0.24, $P=0.01$ ). There was also a significant decrease in hospitalization for worsening CHF with atorvastatin therapy compared with placebo (OR 0.30, $P<0.001$ ).

Conclusions: This meta-analysis suggests the effectiveness of atorvastatin treatment in reducing the risks of all-cause mortality and worsening CHF hospitalization in patients with CHF. Further large, well-conducted randomized trials are needed to confirm the benefits of atorvastatin or other statins for CHF relative to placebo or rosuvastatin.

Key words: statin, atorvastatin, heart failure

\section{Introduction}

The 3-hydroxy-3-methylglutaryl coenzyme A reductase inhibitors (statins) reduce cardiovascular morbidity and mortality in patients with coronary artery disease (CAD) (secondary prevention), and in individuals at high risk of developing CAD (primary prevention) [1, 2]. However, the potential benefits of different statins in patients with chronic heart failure (CHF) remain controversial. Two recent large clinical trials have specifically tested the effect of rosuvastatin in the CHF population, but both yielded disappointing results $[3,4]$. Whether these results of rosuvastatin studies could be extended to the statin class or other statins is unclear. With regards to atorvastatin, the results of several small randomized controlled trials (RCTs) comparing it with placebo in patients with CHF have been reported [5-19], but sample-size limitations in these studies prevented any reliable conclusion. Meta-analysis has the potential to increase the power

\author{
Corresponding author: \\ Minwen Xu, MD \\ Department of Cardiology \\ Huzhou Central Hospital \\ 198 Hongqi Road, Huzhou \\ Zhejiang 313000, China \\ Phone: 865722023301 \\ Fax: 865722023219 \\ E-mail: xuminwen@163.com
}


and improve the precision of treatment effects [20]. Therefore, we performed a meta-analysis based on all currently available RCTs to evaluate the clinical efficacy of atorvastatin in patients with CHF.

\section{Material and methods}

\section{Data sources and selection criteria}

To identify relevant trials, the electronic databases (PubMed, EMBASE and the Cochrane Central Register of Controlled Trials) were searched using the following key words: randomized, statin, hydroxymethylglutaryl-CoA reductase inhibitor, atorvastatin, heart failure, cardiac dysfunction. In addition, we scanned conference proceedings from the American College of Cardiology, the American Heart Association, and the European Society of Cardiology. The search was restricted to articles indexed as a clinical trial involving human subjects. The last search was performed in January 2010.

To be selected for this meta-analysis, studies comparing atorvastatin versus placebo treatment in patients with CHF had to be randomized and have their results reported or made available by the trial investigators. All studies meeting the requirements, regardless of the language or form of publication, were considered to be eligible for this meta-analysis. When there were multiple reports from the same trial, we used the most complete and/or recently reported data.

\section{Study outcomes and data abstraction}

The primary end point was all-cause mortality. Other clinical outcomes of interest included cardiovascular mortality, sudden cardiac death and worsening hospitalization for CHF. Two investigators independently performed data abstraction. In addition to pertinent data on the outcomes of interest, we gathered information on trial names, first author, year of publication, and number of patients enrolled. Disagreements were resolved by consensus.

\section{Statistical analysis}

Data were managed according to the intentionto-treat principle. Odds ratios (OR) with 95\% confidence intervals $(\mathrm{Cl})$ were calculated as summary statistics. The pooled OR was calculated with the Mantel-Haenszel method for fixed effects and the DerSimonian and Laird method for random effects [21, 22]. To assess heterogeneity across trials, we used Cochran's test and means of the $\mathrm{I}^{2}$ statistic [23]. We assessed publication bias with respect to the primary outcomes of interest using a funnel plot as well as the adjusted rank correlation test according to the method of Begg and Mazumdar [24]. A sensitivity analysis was performed by assessing the contribution of individual studies to the summary effect estimate with respect to the primary outcomes. This was done by excluding each trial one at a time and computing meta-analysis estimates for the remaining studies. Results were considered significant at $P<0.05$. Statistical analyses were performed with Stata software version 9.0 (Stata Corp, College Station, USA).

\section{Results}

Of the 955 potentially relevant articles initially screened, a total of 15 trials that randomly compared atorvastatin therapy with placebo in patients with CHF were initially identified [5-19]. Eight studies [5-12] were then excluded due to the prospective crossover design or the lack of data on clinical outcomes. Thus, a total of 7 RCTs were finally included in this meta-analysis, involving 540 patients (296 in the atorvastatin group and 244 controls) [13-19]. The main characteristics of these trials are displayed in Table I and the main baseline characteristics of enrolled patients in individual trials are shown in Table II.

The primary endpoint, all-cause mortality, was reported in all 7 trials. As shown in Figure 1, there was a significant decrease in all-cause mortality with atorvastatin therapy compared with placebo by the fixed-effect model (6.4\% vs. $14.8 \%$, OR 0.39 , $95 \% \mathrm{Cl} 0.21-0.71, P=0.002)$. There was no significant heterogeneity between trials $(P=0.58$, $12=0 \%)$. No evidence of publication bias with respect to all-cause mortality was found using the Begg funnel plot and rank correlation test $(P=1.0)$. Omission of individual trials from the analysis did not have any relevant influence on the overall results.

Both cardiovascular mortality and sudden cardiac death were reported in 5 trials. During the followup period, the risks of cardiovascular mortality (5.5\% vs. $16.8 \%$, OR $0.28,95 \% \mathrm{Cl} 0.12-0.63$, $P=0.002 ; P=0.63, I^{2}=0 \%$ for heterogeneity) and sudden cardiac death $(2.4 \%$ vs. $10.1 \%$, OR 0.24 , $95 \% \mathrm{Cl} 0.08-0.71, P=0.01 ; P=0.75, \mathrm{I}^{2}=0 \%$ for heterogeneity) in patients with atorvastatin therapy were also significantly lower than those in the controls by the fixed-effect model.

Hospitalization for worsening CHF was reported in 6 trials. As shown in Figure 2, there was a significant decrease in hospitalization for worsening CHF with atorvastatin therapy compared with placebo by the fixed-effect model (10.4\% vs. $25.4 \%$, OR $0.30,95 \% \mathrm{Cl} 0.18-0.52, P<0.001)$. There was no significant heterogeneity between trials $\left(P=0.76, I^{2}=0 \%\right)$.

\section{Discussion}

By pooling all currently available RCTs which reported clinical effects of atorvastatin in patients 


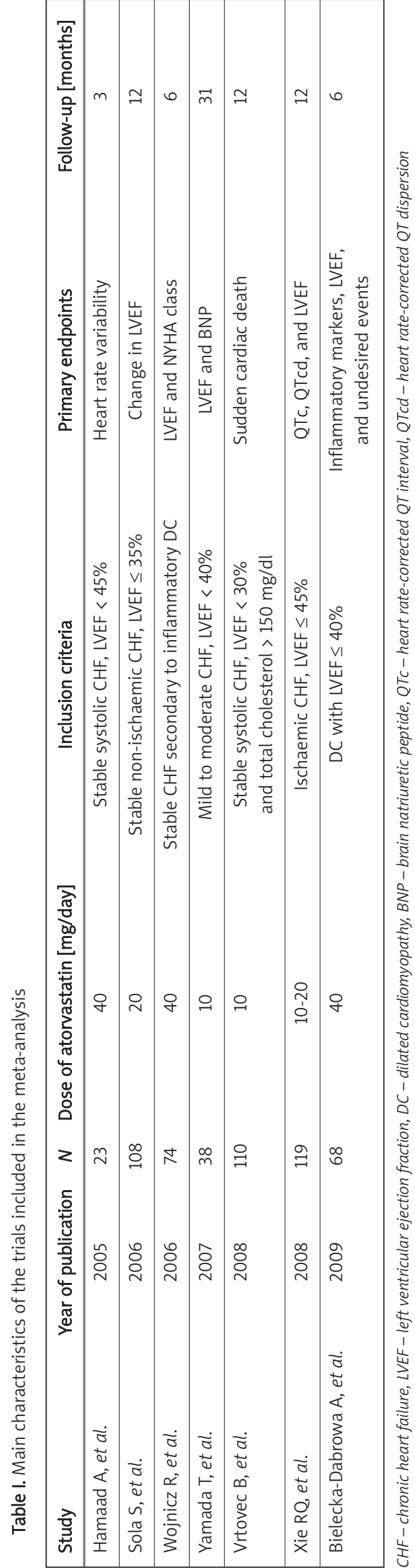

with $\mathrm{CHF}$, the present meta-analysis showed that atorvastatin was associated with significantly decreased risks of all-cause mortality, cardiovascular mortality, sudden cardiac death and hospitalization for worsening CHF, as compared with placebo.

Statins are widely known as therapeutic agents for hypercholesterolaemia. Recently, evidence of their pleiotropic effects, meaning outside of the ability to reduce cholesterol levels, has emerged. The potent pleiotropic effects of statins, including potential benefits for myocardial cellular function, down-regulation of tissue renin-angiotensinaldosterone system activation, restoration of autonomic function, neoangiogenesis, and inhibition of proinflammatory cytokines, could favourably influence the natural history of CHF [25-30]. Statins possess anti-inflammatory effects and decrease vascular cell adhesion molecule-1 levels in CHF.31 Additionally, circulating lipids have the capacity to detoxify bacterial lipopolysaccharides, which stimulate the release of inflammatory cytokines in CHF [32]. Taken together, the rationale supporting the potential benefits of statin therapy in $\mathrm{CHF}$ is well grounded [33, 34].

The first indirect information about the beneficial effects of statins in CHF came from the post hoc analyses of a landmark clinical trial of statin therapy. In the Scandinavian Simvastatin Survival Study (4S) [35], patients who had known CAD and hypercholesterolaemia were included. A lower incidence of symptomatic CHF was observed in the simvastatin group, compared with the placebo group. Also, there was a $19 \%$ relative risk reduction in 5 -year mortality with statin therapy in patients who had CHF. Additionally, the Long-Term Intervention with Pravastatin in Ischaemic Disease (LIPID) [36] trial observed a mortality benefit of pravastatin in CHF $(P<0.05)$, while the Heart Protection Study (HPS) [37] showed a marginal reduction in mortality (3.4\% in simvastatin vs. 3.9\% in placebo group) and hospitalization. The A to $Z$ trial [38] showed reduction of new-onset CHF by $18 \%(P<0.05)$.

Recently, data from large observational studies were consistent in showing a beneficial effect of statins in patients with CHF [39-44]. The retrospective trial by Foody et al. [39] evaluated statin therapy in patients admitted to acute care hospitals with a principal diagnosis of CHF. A sample of 54960 patients, all more than 65 years of age, was analysed. Patients who received statins on discharge $(16.7 \%)$ were shown to have significant reductions in 1-year mortality by $20 \%(P<0.001)$ and 3-year mortality by $18 \%(P<0.001)$, even after adjustment for clinical confounders. However, these patients also had higher rates of cardiac investigations, were more likely to be seen by a cardiologist and received a higher rate of 


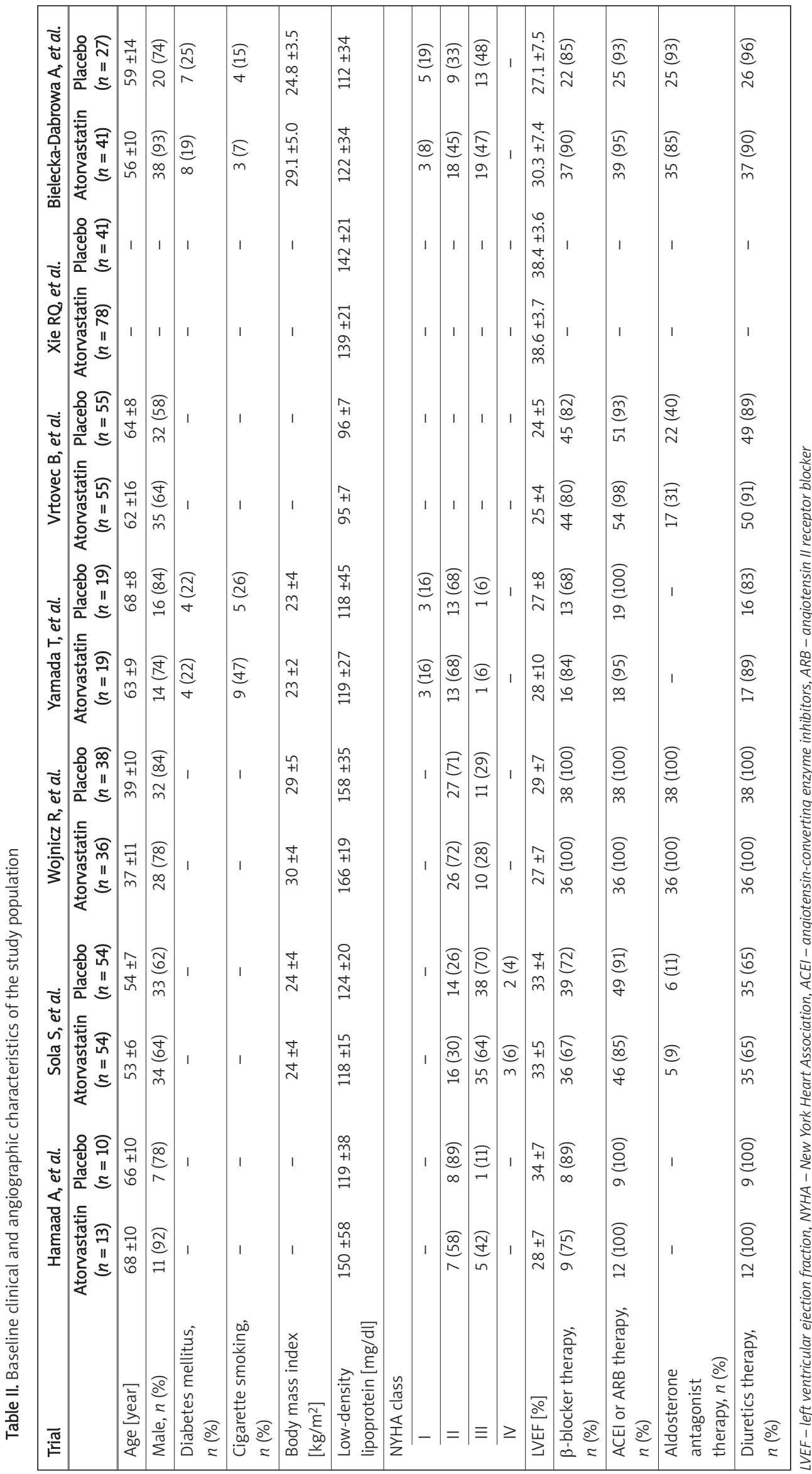




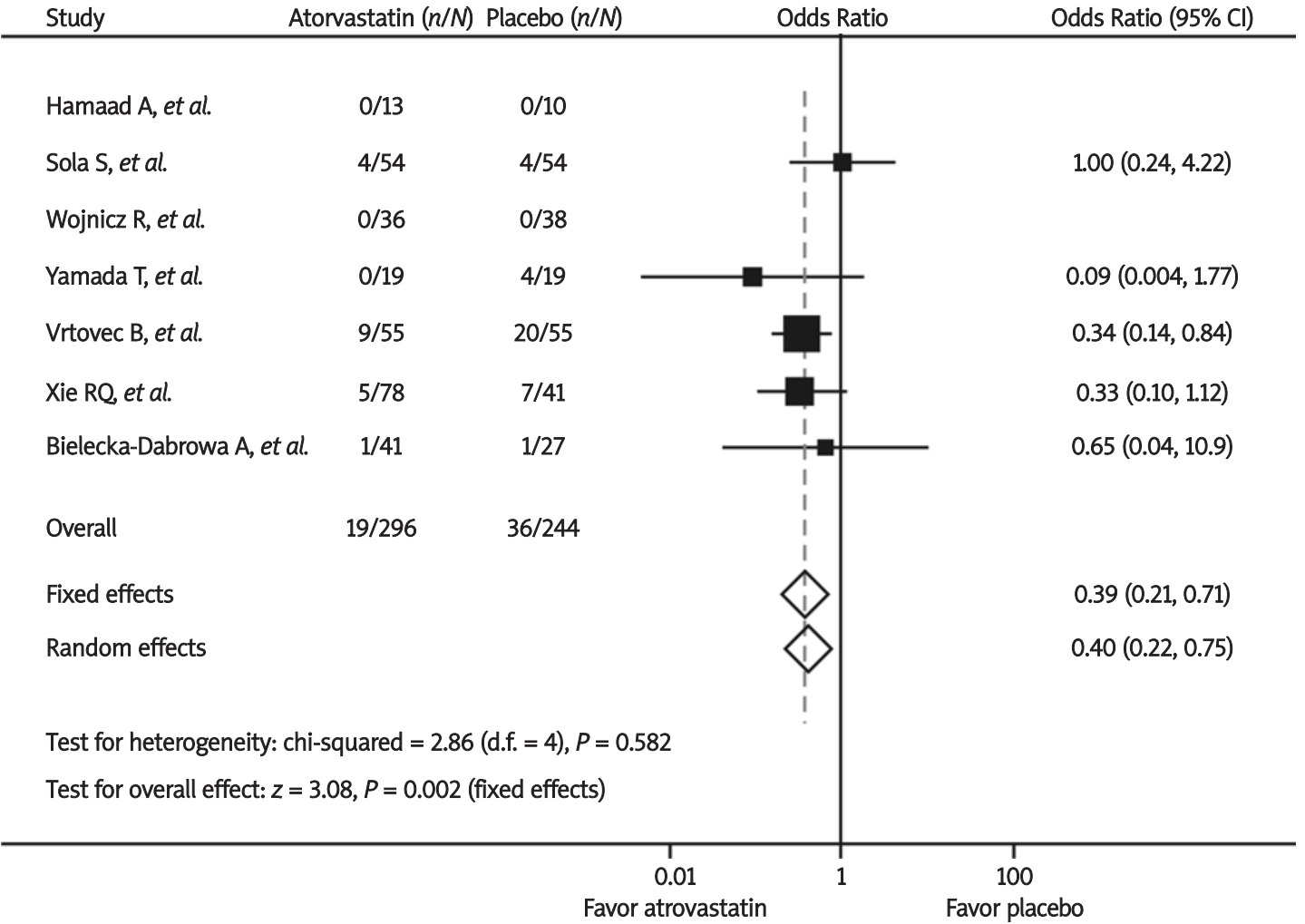

Figure 1. Odds ratios of all-cause mortality associated with atorvastatin versus placebo from individual studies and overall population. The size of the data markers (squares) is approximately proportional to the statistical weight of each trial

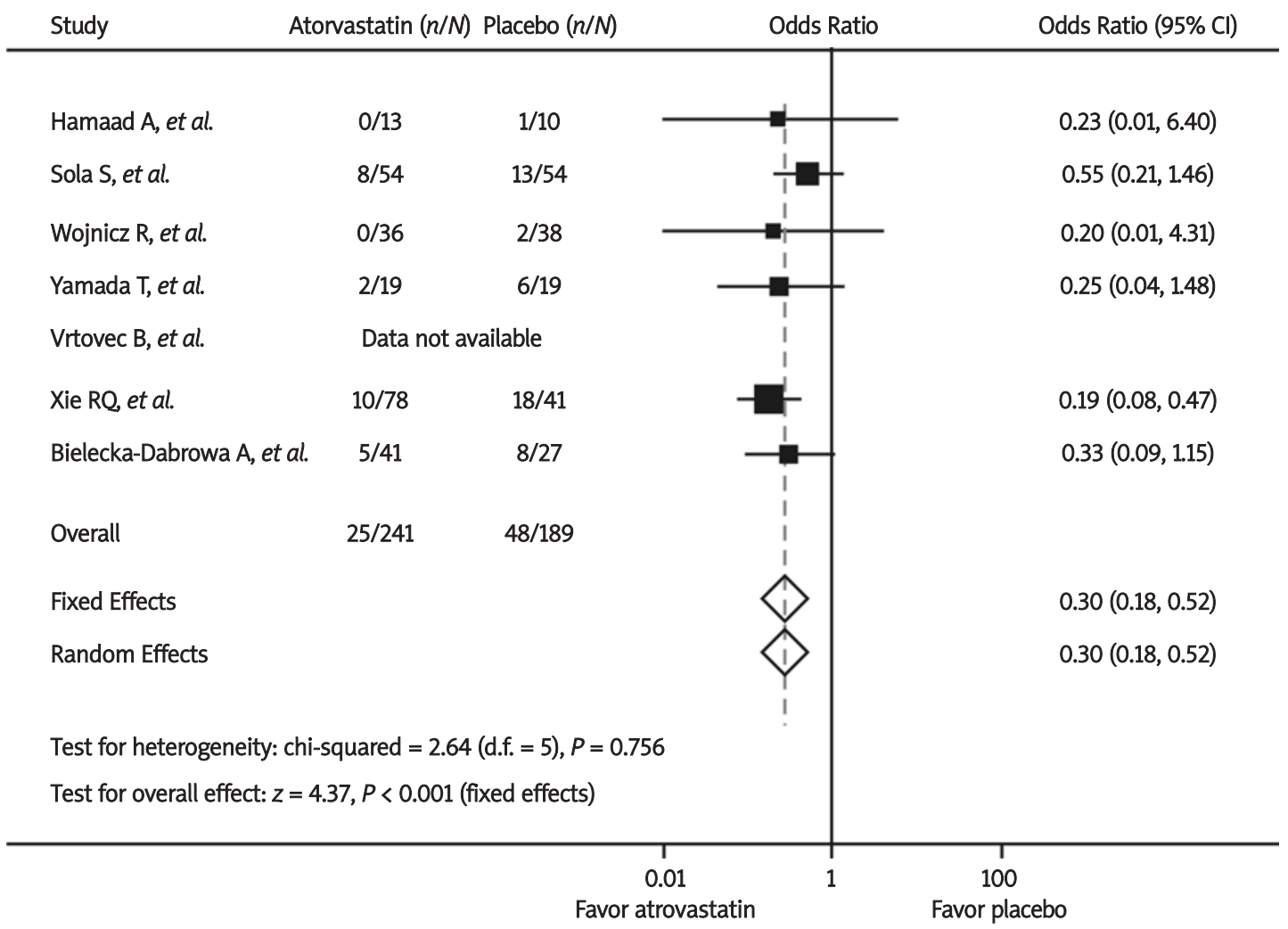

Figure 2. Odds ratios of hospitalization for worsening chronic heart failure associated with atorvastatin versus placebo from individual studies and overall population. The size of the data markers (squares) is approximately proportional to the statistical weight of each trial 
concomitant aspirin, beta-blockers and angiotensinconverting enzyme inhibitor prescriptions on discharge. Another large observational cohort study by Go et al. [40] involved 24598 adults diagnosed with CHF who had no prior statin use. Using an intent-to-treat approach, incident statin use was associated with a $21 \%(P<0.001)$ and $24 \%$ $(P<0.001)$ reduction in hospitalization for $\mathrm{CHF}$ and total mortality, respectively. It is important to note that statins were more likely to be prescribed in younger patients and those known to have CAD, diabetes, or hypertension in this study. A prespecified post hoc analysis of the TNT trial (80 vs. $10 \mathrm{mg} / \mathrm{d}$ atorvastatin, $n=10001$ with (AD) showed that intensive treatment with atorvastatin in patients with stable coronary disease significantly reduces subsequent hospitalizations for $\mathrm{CHF}$ compared with low-dose therapy (2.4\% vs. $3.3 \%$, $P=0.0116$ ) [43]. Moreover, this benefit was most pronounced in patients with a history of CHF (41\% lower, $P=0.008$ ), while in patients without a history of $\mathrm{CHF}$, hospitalization rates for $\mathrm{CHF}$ were similar in the high- and low-dose atorvastatin groups.

Contrary to the previously mentioned reports of benefit with statin therapy, two large, prospective randomized trials with robust clinical end points present conflicting data. The CORONA trial [3] assessed the effectiveness and safety of rosuvastatin $10 \mathrm{mg}$ versus placebo in addition to optimal CHF treatment in prevalent symptomatic CHF cases, with a history of myocardial infarction and not previously treated with a statin. The study reported no effect on all-cause mortality and hospitalization over the median follow-up period of 32 months (HR 0.95; $P=0.31$ ), though safety of the statin was demonstrated. With regards to the reasons why CORONA did not show the expected benefits, one possibility is the study population (CORONA included elderly subjects [mean age 73 years; $41 \%$ were older than 75 years] with severe systolic CHF, and $61 \%$ of the CHF population was New York Heart Association [NYHA] class III with a mean ejection fraction of $31 \%$ ). It is possible that rosuvastatin was administered too late in the natural course of CHF to show a benefit. Another explanation is that patients in the CORONA study were receiving almost optimal background treatment for CHF (most patients were on a reninangiotensin-aldosterone system [RAAS] blocker [91\%], a loop or thiazide diuretic [89\%], a betablocker [75\%], and antiplatelet or anticoagulant therapy [90\%]). Therefore, it might be that the CORONA population was "too well treated" to achieve an additional benefit from statins. The GISSI-HF trial [4] enrolled patients aged 18 years or older with symptomatic CHF of ischaemic and nonischaemic cause, randomly assigned them to rosuvastatin $10 \mathrm{mg}$ daily or placebo, and followed them up for a median of 3.9 years. No effect was seen in all-cause mortality or combined mortality and hospital admissions (HR 1.0 and 1.1, respectively). The GISSI-HF trial differed from the CORONA trial in some aspects of the population enrolled (lower proportion with ischaemic aetiology, younger age, and less severe symptoms). However, patients in the GISSI-HF study were also receiving optimal background treatment for $\mathrm{CHF}$ and might be "too well treated" to achieve an additional benefit from statins. Moreover, it remains possible that benefits of statins may be offset by a negative impact of cholesterol lowering in CHF patients, as some evidence suggests that low serum total cholesterol is associated with a marked increase in mortality in advanced CHF.

Although there is overwhelming evidence that statins are equally effective for primary and secondary prevention of cardiovascular disease, statins may differ in their properties in patients with CHF and most of the studies with favourable outcomes used drugs other than rosuvastatin. Likewise, the present meta-analysis found that atorvastatin could significantly decrease risks of allcause mortality, cardiovascular mortality, sudden cardiac death and hospitalization for worsening symptoms as compared to placebo in patients with CHF. One of the differences between atorvastatin and rosuvastatin is lipophilicity. Atorvastatin is more lipophilic than rosuvastatin and may penetrate the cell membrane more effectively, which could provide a theoretical advantage [45]. Moreover, lipophilic statins may be superior to hydrophilic statins in preventing cardiovascular events because of their potent pleiotropic effects such as the inhibition of pro-inflammatory cytokine production or matrix metalloproteinase expression [46, 47]. These results suggested that different statins may have inconsistent benefits regarding important clinical consequences of CHF. Thus, the fact that a unique statin agent (rosuvastatin) was used in CORONA and GISSI-HF trials might be one of the causes of their negative results, which could not be automatically extrapolated to other statins.

Our results suffer from those limitations which are inherent to all meta-analytic techniques, including particularly heterogeneity in patient populations, different study drug regimens, and variable endpoint definitions and follow-up periods across studies. In particular, in this analysis we used aggregate data as reported or calculated in published articles, rather than data of individual patients. Furthermore, although there was no statistical heterogeneity in our analysis, the number of patients is very small overall and also in each trial, and the number of events is very small. Given the mean small sample size of the studies included 
in our study, our findings should be interpreted with caution. Further large, well-conducted randomized trials are needed to confirm the benefits of atorvastatin or other statins for $\mathrm{CHF}$ relative to placebo or rosuvastatin.

\section{References}

1. Baigent C, Keech A, Kearney PM, et al.; Cholesterol Treatment Trialists' (CTT) Collaborators. Efficacy and safety of cholesterol-lowering treatment: prospective metaanalysis of data from 90,056 participants in 14 randomised trials of statins. Lancet 2005; 366: 1267-78.

2. Mills EJ, Rachlis B, Wu P, Devereaux PJ, Arora P, Perri D. Primary prevention of cardiovascular mortality and events with statin treatments: a network meta-analysis involving more than 65,000 patients. J Am Coll Cardiol 2008; 52: 1769-81.

3. Kjekshus J, Apetrei E, Barrios V, et al; CORONA Group. Rosuvastatin in older patients with systolic heart failure. N Engl J Med 2007; 357: 2248-61.

4. Gissi-HF Investigators, Tavazzi L, Maggioni AP, Marchioli $R$, et al. Effect of rosuvastatin in patients with chronic heart failure (the GISSI-HF trial): a randomised, doubleblind, placebo-controlled trial. Lancet 2008; 372: 1231-9.

5. Tousoulis D, Antoniades C, Bosinakou E, et al. Effects of atorvastatin on reactive hyperemia and inflammatory process in patients with congestive heart failure. Atherosclerosis 2005; 178: 359-63.

6. Strey CH, Young JM, Molyneux SL, et al. Endotheliumameliorating effects of statin therapy and coenzyme Q10 reductions in chronic heart failure. Atherosclerosis 2005; 179: 201-6.

7. Tousoulis D, Antoniades C, Vassiliadou C, et al. Effects of combined administration of low dose atorvastatin and vitamin $\mathrm{E}$ on inflammatory markers and endothelial function in patients with heart failure. Eur J Heart Fail 2005; 7: 1126-32.

8. Mozaffarian D, Minami E, Letterer RA, Lawler RL, McDonald GB, Levy WC. The effects of atorvastatin (10 $\mathrm{mg}$ ) on systemic inflammation in heart failure. Am J Cardiol 2005; 96: 1699-704.

9. Vrtovec B, Okrajsek R, Golicnik A, Ferjan M, Starc V, Radovancevic B. Atorvastatin therapy increases heart rate variability, decreases QT variability, and shortens QTC interval duration in patients with advanced chronic heart failure. J Card Fail 2005; 11: 684-90.

10. Bleske BE, Nicklas JM, Bard RL, et al. Neutral effect on markers of heart failure, inflammation, endothelial activation and function, and vagal tone after high-dose HMG-CoA reductase inhibition in non-diabetic patients with non-ischemic cardiomyopathy and average low-density lipoprotein level. J Am Coll Cardiol 2006; 47: 338-41.

11. Strey CH, Young JM, Lainchbury JH, et al. Short-term statin treatment improves endothelial function and neurohormonal imbalance in normocholesterolaemic patients with non-ischaemic heart failure. Heart 2006; 92: 1603-9.

12. Young JM, Strey $\mathrm{CH}$, George PM, et al. Effect of atorvastatin on plasma levels of asymmetric dimethylarginine in patients with non-ischaemic heart failure. Eur J Heart Fail 2008; 10: 463-6.

13. Hamaad A, Sosin M, Lip GY, MacFadyen RJ. Short-term adjuvant atorvastatin improves frequency domain indices of heart rate variability in stable systolic heart failure. Cardiovasc Drugs Ther 2005; 19: 183-7.
14. Sola S, Mir MO, Lerakis S, Tandon N, Khan BV. Atorvastatin improves left ventricular systolic function and serum markers of inflammation in nonischemic heart failure. J Am Coll Cardiol 2006; 47: 332-7.

15. Wojnicz R, Wilczek K, Nowalany-Kozielska E, et al. Usefulness of atorvastatin in patients with heart failure due to inflammatory dilated cardiomyopathy and elevated cholesterol levels. Am J Cardiol 2006; 97: 899-904.

16. Yamada T, Node K, Mine T, et al. Long-term effect of atorvastatin on neurohumoral activation and cardiac function in patients with chronic heart failure: a prospective randomized controlled study. Am Heart J 2007; 153: 1055.e1-e8.

17. Vrtovec B, Okrajsek R, Golicnik A, et al. Atorvastatin therapy may reduce the incidence of sudden cardiac death in patients with advanced chronic heart failure. J Card Fail 2008; 14: 140-4.

18. Xie RQ, Cui W, Liu F, Yang C, Pei WN, Lu JC. Statin therapy shortens QTC, QTcd, and improves cardiac function in patients with chronic heart failure. Int J Cardiol 2010; 140: 255-7.

19. Bielecka-Dabrowa A, Goch JH, Mikhailidis DP, Rysz J, Maciejewski M, Banach $M$. The influence of atorvastatin on parameters of inflammation and function of the left ventricle in patients with dilated cardiomyopathy. Med Sci Monit 2009; 15: MS12-23.

20. Egger M, Ebrahim S, Smith GD. Where now for metaanalysis? Int J Epidemiol 2002; 31: 1-5.

21. Mantel N, Haenszel W. Statistical aspects of the analysis of data from retrospective studies of disease. J Natl Cancer Inst 1959; 22: 719-48.

22. DerSimonian R, Laird N. Meta-analysis in clinical trials. Control Clin Trials 1986; 7: 177-88.

23. Higgins JP, Thompson SG, Deeks JJ, Altman DG. Measuring inconsistency in meta-analyses. BMJ 2003; 327: 557-560.

24. Begg CB, Mazumdar M. Operating characteristics of a rank correlation test for publication bias. Biometrics 1994; 50: 1088-101.

25. Blum A, Shamburek R. The pleiotropic effects of statins on endothelial function, vascular inflammation, immunomodulation and thrombogenesis. Atherosclerosis 2009; 203: 325-30.

26. Calabrò P, Yeh ET. The pleiotropic effects of statins. Curr Opin Cardiol 2005; 20: 541-6.

27. Zhang F, Dong L, Ge J. Effect of statins pretreatment on periprocedural myocardial infarction in patients undergoing percutaneous coronary intervention: a metaanalysis. Ann Med 2010; 42: 171-7.

28. Angelopoulos J, Krassakopoulos N, Nathanson R, Boukas $\mathrm{S}$, Sampalis JS. Co-administration of ezetimibe and a statin in management of dyslipidemias: a meta-analysis of clinical trials. Arch Med Sci 2009; 5: 347-63.

29. Wainwright G, Mascitelli L, Goldstein MR. Cholesterollowering therapy and cell membranes. Stable plaque at the expense of unstable membranes? Arch Med Sci 2009; 5: 289-95.

30. Ravipati G, Aronow WS, Kumbar S, et al. Patients with diabetes mellitus with ischemic stroke have a higher hemoglobin Alc level and a higher serum low-density lipoprotein cholesterol level than diabetics without ischemic stroke. Arch Med Sci 2009; 5: 391-3.

31. Nachtigal P, Jamborova G, Pospisilova N, et al. Atorvastatin has distinct effects on endothelial markers in different mouse models of atherosclerosis. J Pharm Pharm Sci 2006; 9: 222-30.

32. Rauchhaus M, Coats AJ, Anker SD. The endotoxinlipoprotein hypothesis. Lancet 2000; 356: 930-3. 
33. Mathur N, Ramasubbu K, Mann DL. Spectrum of pleiotropic effects of statins in heart failure. Heart Fail Clin 2008; 4: 153-61.

34. Ramasubbu K, Estep J, White DL, Deswal A, Mann DL. Experimental and clinical basis for the use of statins in patients with ischemic and nonischemic cardiomyopathy. J Am Coll Cardiol 2008; 51: 415-26.

35. Kjekshus J, Pedersen TR, Olsson AG, Faergeman O, Pyörälä $K$. The effects of simvastatin on the incidence of heart failure in patients with coronary heart disease. J Card Fail 1997; 3: 249-54.

36. The Long-Term Intervention with Pravastatin in Ischaemic Disease (LIPID) Study Group. Prevention of cardiovascular events and death with pravastatin in patients with coronary heart disease and a broad range of initial cholesterol levels. N Engl J Med 1998; 339: 1349-57.

37. Heart Protection Study Collaborative Group. The effects of cholesterol lowering with simvastatin on cause-specific mortality and on cancer incidence in 20,536 high-risk people: a randomised placebo-controlled trial. BMC Med 2005; 3: 6.

38. de Lemos JA, Blazing MA, Wiviott SD, et al.; A to $Z$ Investigators. Early intensive vs a delayed conservative simvastatin strategy in patients with acute coronary syndromes: phase $Z$ of the A to $Z$ trial. JAMA 2004; 292: 1307-16.

39. Foody JM, Shah R, Galusha D, Masoudi FA, Havranek EP, Krumholz HM. Statins and mortality among elderly patients hospitalized with heart failure. Circulation 2006; 113: 1086-92.

40. Go AS, Lee WY, Yang J, Lo JC, Gurwitz JH. Statin therapy and risks for death and hospitalization in chronic heart failure. JAMA 2006; 296: 2105-11.

41. Ray JG, Gong Y, Sykora K, Tu JV. Statin use and survival outcomes in elderly patients with heart failure. Arch Intern Med 2005; 165: 62-7.

42. Scirica BM, Morrow DA, Cannon CP, et al. Intensive statin therapy and the risk of hospitalization for heart failure after an acute coronary syndrome in the PROVE IT-TIMI 22 study. J Am Coll Cardiol 2006; 47: 2326-31.

43. Khush KK, Waters DD, Bittner V, et al. Effect of high-dose atorvastatin on hospitalizations for heart failure: subgroup analysis of the Treating to New Targets (TNT) study. Circulation 2007; 115: 576-83.

44. Node K, Fujita M, Kitakaze M, Hori M, Liao JK. Short-term statin therapy improves cardiac function and symptoms in patients with idiopathic dilated cardiomyopathy. Circulation 2003; 108: 839-43.

45. Schachter, M. Chemical, pharmacokinetic and pharmacodynamic properties of statins: an update. Fundam. Clin Pharmacol 2005; 19: 117-25.

46. Ikeda U, Shimpo M, Ohki R, et al. Fluvastatin inhibits matrix metalloproteinase- 1 expression in human vascular endothelial cells. Hypertension 2000; 36: 325-9.

47. Ito T, Ikeda U, Yamamoto K, Shimada K. Regulation of interleukin-8 expression by HMG-CoA reductase inhibitors in human vascular smooth muscle cells. Atherosclerosis 2002; 165: 51-5. 